ploration may proceed are numerous and attractive. We have only to follow the example set by Laplace, Poisson, Green, Gauss, Maxwell, Kirchhoff, Saint-Venant, Helmholtz, and their eminent contemporaries and successors. In commending the works of these great masters, to the younger members especially of the American Mathematical Society, I would not be understood as urging the cultivation of pure mathematics less, but rather as suggesting the pursuit of applied mathematics more. The same sort of fidelity to research and the same sort of genius for infinite industry which enabled those masters to accomplish the grand results of the nineteenth century may be confidently expected to achieve equally grand results in the twentieth century.

\title{
THE STATUS OF IMAGINARIES IN PURE GEOMETRY.
}

(Read before the American Mathematical Society, October 28, 1899.)

IN teaching the elements of analytical geometry we are practically forced to allow, even to encourage, a slipshod identification of the field of geometry with the field of algebra. We must all have realized the disadvantages attendant on this course. If ever we have the chance of repairing the error-if error indeed it be at that stage-it is in teaching synthetic geometry; but we can repair it then only if we can establish the existence of imaginary elements without the slightest dependence on algebra. Many books refer to the analogy of algebra as affording sufficient basis, others openly rely on algebraic principles; Chasles, for instance, in the Géométrie Supérieure (pp. 54-57) relies essentially on quadratic equations, whose imaginary roots assure him of the existence of imaginary points.

The two chief books that deal with absolutely pure geometry are those of von Staudt and Reye. It is one of the axioms of modern mathematics that von staudt placed the doctrine of imaginaries on a firm geometrical basis; but logical and convincing as his treatment is, when patiently studied in all its detail, it yet seems to me hardly practicable as a class-room method.

Von Staudt's primary domain is the visible universe; the elements of his geometry, together with the idea of direction, are an intellectual abstraction from the results of observation. He then extends his domain beyond the visible universe by formal definition; to replace the idea of direction he introduces a set of "ideal points," and 
finds that the nature of an ideal point is the same as that of a common point, and that the relation of the ideal points and lines to one another is precisely that of points and lines in a plane. Thus his second domain is the visible universe increased by one ideal plane.

To attain absolute consistency of language he introduces pairs of feigned, or imaginary, elements. Certain arrangements of pairs of points on a line give rise to a pair of points ; certain other arrangements, differing only in their pictured form, apparently do not. Now as we have already extended the domain of geometry beyond the visible universe, the fact that the point pair is not visible cannot be accepted as a proof that it is non-existent. Although the conception of the range of points with its contents is derived from observation, yet we have to take into account the fact that we may not be able to observe the whole. Hence instead of jumping to the conclusion that the points are nonexistent, we investigate the alternative hypothesis, namely, that there are still two points, though not pictured. As regards this purely intellectual hypothesis we have to enquire whether it is tenable, and whether it is manageable.

To prove that the hypothesis is tenable, von Staudt had first to find some device for separating the two elements of a pair, seeing that his geometry accepts a point as element. not a point pair. This difficulty delayed him from 18471856 ; he overcame it finally by a sublimated intellectual artifice, that of the "sense" of a form. He had then to show that every feigned, or imaginary, element is endowed with every formal property of a natural element. His feigned elements are (1) imaginary points, each lying by definition on one real straight line (imaginary elements of a range); (2) imaginary planes, each passing by definition through one real straight line (imaginary elements of an axial pencil); (3) imaginary lines of the first kind, each lying by definition in one real plane and passing through one real point (imaginary elements of a flat pencil); (4) imaginary lines of the second kind, each belonging by definition to a real regulus, but possessing no real point and no real plane. He investigates every possible relation of real and imaginary points, lines, and planes, defining with great care every phrase that has reference to the imaginary elements. Of course all this detail fills up page after page, even unto weariness ; but by it he does prove that adopting the particular phraseology that assumes the existence of these feigned elements, all verbal consequences are correct. Thus the hypothesis is shown to be tenable. 
Von Staudt states explicitly (Beiträge, p. 77) that there are no other imaginary elements; that is to say, that no use of the accepted geometrical processes will lead us outside the domain now defined. He shows also that correlating the real (or observed) elements of two forms does also correlate the imaginary (or inferred) elements. Thus there is no danger of unknown regions of imaginaries opening up before us ; we have extended the domain, it is true, but not indefinitely. To complete the proof that the hypothesis is manageable, we must next investigate the nature of this extended domain, in order to determine whether the imaginary elements, thus separately defined, are arranged in accordance with any law of continuity. Von Staudt's results prove, for example, as regards an imaginary straight line of the first or second kind, that the totality of points can be pictured on a real plane, and that there is the same possibility of passing from one point to another as from one point of the plane to another.

Thus the hypothesis is both tenable and manageable; by introducing these formal elements he enlarges the domain of his geometry, without in any way affecting its nature or modifying its laws.

The principal objections to this, as an elementary method, are the following:-

(1) the extreme difficulty and tediousness of the complete proof of the identity of the properties of natural elements and formal elements.

(2) the apparent break in passing from the seen to the unseen, which arouses scepticism as to whether the formal elements can truly be said to "exist." While the reason, if sufficiently trained, is convinced, all natural instincts rebel. The whole thing impresses the natural man as simply a tour de force.

We turn now to geometry as treated by Reye; or rather, as it would naturally have been treated by Reye if he had carried out the plan of his first chapter.* Elements of threekinds are given, absolutely- $a, b$ and $c$-elements (points, lines, and planes), with a statement of properties and relations to serve instead of definitions. Attaching to these elements the numbers $1,2,3$, and working with modulus 4 , a number of the relations can be obtained arithmetically ; for example, $1+1=2$ expresses that two points determine a line; $3+3=6=2$ [mod. 4$]$, two planes determine a line ; sim-

* As I interpret it ; it appears however that some others find in it a much closer agreement with the fundamental conceptions of von Staudt. 
ilarly $1+1+1=3,3+3+3=9=1[\bmod .4], 1+2=3$, $3+2=5=1$ [mod. 4] give certain of the relations; $2+2=4=0$ [mod. 4 ] expresses the fact that two lines determine nothing. Multiplication by -1 reciprocates; $1+2=3$ becomes $-1+(-2)=-3$, that is, $3+2=1$ [mod. 4]. A trivial suggestion, perhaps; but it brings out clearly the fact that the elements $a, b, c$ are not precisely defined as points, lines, and planes.

Reye now makes a very arbitrary assumption, namely, that the elements can be identified with the points, lines, and planes of ordinary space. This identification gives the points at infinity, to account for the disappearing intersection of lines in a plane; it gives rise also to such assumptions as that a line and a conic in a plane may possibly not intersect. Finally, imaginary elements are introduced, with a reference to von Staudt for the justification.

Now as I read Reye's first chapter, in the logical development of the system infinitely distant and imaginary elements must be simply a result of classification. We are given all points, all lines, all planes; that is all $a, b$, and c-elements that can have certain relations to one another, with certain properties that hold without exception. Then we observe that points, lines, and planes in the visible universe illustrate, or picture, these properties on the whole; and that we can make the picturing more exact, in detail, if we regard parallel lines as meeting somewhere. In fact, this is a necessary condition of the picturing ; for if two parallel lines do not meet, they cannot represent two $b$-elements that lie in a c-element. Thus the conception of infinitely distant elements arises from the attempt to picture this particular intellectual domain in the visible universe. From this attempt there arises also the distinction between real and imaginary elements. For while the visible points, lines, and planes serve to picture some of the $a, b, c$-elements, we have no right to assume that they suffice to picture all. Allowing for this, we divide elements into picturable and non-picturable, and examine whether there are any in the second division.

This point cannot be decided until we make some additional statement about the nature of the given elements; perhaps it is enough to affirm the indestructibility of the elements of any given space, whether the elements be given singly and explicitly, or by implication as determined by other elements. Thus, for example, two lines in a plane determine a point, and no rotation of either line can cause this point to pass out of existence. Two point pairs $A, A^{\prime}$; 
$B, B^{\prime}$ on a line are proper for the determination of a third point pair $C, C^{\prime}$, namely, the common harmonic pair ; the motion of one element $B^{\prime}$ along the given line causes a change in the positions of $C, C^{\prime}$, but it cannot cause these points to pass out of existence; the common harmonic pair is determinate, however the two pairs $A, A^{\prime} ; B, B^{\prime}$ may be placed. Now picturing the two pairs on a line, it is found that if the segments $A, A^{\prime} ; B, B^{\prime}$ overlap, the third pair is non-picturable. Hence there are elements in the second division ; and the only ones that we have detected occur by pairs.

Thus to construct this purely formal geometry we postulate elements of three kinds, $a, b$, $c$, with certain relations, in which most of their properties are formulated. In addition to the numerical relations already mentioned, we have to state that any one element can move in any other, adding explicitly that an $a$ or $c$ moving in a $b$ can move only "forward" or "backward," and that elements are indestructible. At any stage we may attempt to picture these formal elements by means of a set of existing elements, subject to the same laws of combination, and may thus obtain a division of our formal elements into those that can be pictured in this way, and those that cannot; the division into real and imaginary results from the attempt to picture these formal elements in ordinary space.

This is merely intended as a suggestion to practical teachers; it is not set forth as a fully worked out scheme. A different formulation of the relations and properties may be preferable, but I believe the principle to be sound. If the elements are given in a purely intellectual manner, and not as abstractions from observation of the visible universe, then the distinction between real elements on the one hand, imaginary elements on the other, is purely a result of classification; the imaginary elements are given together with the real ones, instead of being adjoined to them by definition. We can even decide for ourselves that certain selected elements shall be pictured; but to say how many we can choose arbitrarily, and what others will then fall into the picturable class (real class), is hardly possible without considering von Staudt's theory of chains or strands (Ketten). Any exhaustive investigation of the number and arrangement of the picturable and non-picturable elements of any form must ultimately coincide with von Staudt's; but for the mere introduction of imaginaries into ordinary teaching the plan here suggested for avoiding these long discussions seems feasible. It has the additional advantage of justify- 
ing the use of diagrams in proving results that depend on so-called imaginaries.

I may add that in this strictly formal geometry metric relations must of course be introduced independently, by specifying an absolute configuration in the given domain, and classing as metric all relations of any form to this absolute configuration.

Bryn Mawr, Pennsyluania,

Charlotte Angas Scott. October, 1899.

\section{NOTES.}

The presidential address of Professor R. S. Woodward, which appears in the present number of the Bulletis, is also published separately in reprint form. Copies may be obtained from the Secretary at twenty-five cents each.

The advisory board for mathematics at Cambridge University has recommended to the Senate of that institution a number of changes in the regulations relating to the mathematical tripos. It is proposed to omit entirely from Part I. the following subjects:-calculus of variations, elliptic functions, besselian functions, hydrodynamics, and sound, and to limit the extent of the requirements in rigid dynamics, electricity, optics, astronomy, and other subjects. The arrangement of the examination papers is to be changed. No papers are to be devoted exclusively to problems. At least half of the questions set throughout the examination shall be of an elementary character. The classic general restrictions as to methods of solutions for certain papers are to be no longer maintained. The ancient order of merit is to be abandoned. The successful candidates are to be arranged in three classes (wranglers, senior and junior optimes) of three divisions each, the names in each division being placed in alphabetical order. Corresponding changes are proposed for Part II. of the tripos. The Senate returned the proposals to the board for revision.

At one time the largest tripos at Cambridge, the mathematical tripos is now smaller than either the classical or the natural science tripos. For the four years 1869-72, one in eighteen of the resident undergraduates passed the mathematical tripos, Part I.; while for the five years $1895-99$, the average falls to one in thirty-five. 